\title{
Competencias Docentes para una Pedagogía Inclusiva. Consideraciones a partir de la Experiencia con Formadores de Profesores Chilenos
}

\author{
Teacher Competences to Inclusive Pedagogy. Considerations \\ from Chilean Teacher Educators' Experiences
}

\author{
Carolina García González * \\ Constanza Herrera-Seda \\ Carlos Vanegas Ortega
}

Universidad de Santiago de Chile, Chile

\begin{abstract}
La formación de profesores en educación inclusiva constituye una condición clave para avanzar hacia una enseñanza equitativa y de calidad para todo el estudiantado. Esta investigación buscó caracterizar cuán preparados se sienten los formadores de profesores en las competencias necesarias para una educación inclusiva y con qué frecuencia los formadores implementan estrategias de enseñanza y apoyo basadas en la valoración de la diversidad de los estudiantes. A partir de una investigación cuantitativa, de alcance descriptivo y corte transversal, se analizaron las percepciones de 143 docentes chilenos mediante dos escalas de auto-reporte. Se encontró que, en general, estos declaran estar preparados para la formación desde una perspectiva inclusiva, e implementar con frecuencia acciones que contribuyen a garantizar el aprendizaje y la participación de los estudiantes de pedagogía. Asimismo, la investigación permitió identificar algunas dimensiones que deben ser fortalecidas, entre las que destacan la innovación de la docencia y el diseño de experiencias de aprendizaje flexibles, en donde se aproveche el uso de tecnologías. Finalmente, se discute la importancia de contar instrumentos sensibles a la particularidad del quehacer del formador de profesores, así como de programas de perfeccionamiento específicos para el fortalecimiento de su rol desde una perspectiva inclusiva.
\end{abstract}

Descriptores: Formador de docentes; Competencias del docente; Formación de docentes; Educación inclusiva.

Teacher training in inclusive education is a key condition to moving towards equity and quality teaching for all students. This research aims to characterize how prepared the teachers educators perceived they were in the needed competences of inclusive education and how often they performed teaching and support strategies based on appreciation of student diversity. As from a quantitative research, crosssectional with a descriptive scope, the perception of 143 Chilean teacher educators was analyzed through two self-reported scales. In general, it was found they feel prepared to train from an inclusive perspective, and frequently implemented actions that contributed to guarantee the learning and participation of student teachers. Likewise, this research allows us to identify some dimensions that should be strengthened, among which stand the teaching innovation and the design of flexible learning experiences that take advantage of the use of technology. Finally, the relevance of having instruments with sensitivity to peculiarity of teacher educator work and specific training programs to firm up their role in an inclusive perspective are discussed.

Keywords: Teacher educators; Teacher qualifications; Teaching education; Inclusive education. 


\section{Introducción}

En las últimas décadas, el avance hacia una educación inclusiva se ha transformado en un imperativo ético y legal, pues se ha arribado a la comprensión de que la equidad de los sistemas educativos constituye un requisito para la calidad (Marchesi y Martín, 2014). En este sentido, la Agenda 2030 para el Desarrollo Sostenible definida por UNESCO (2015) plantea que uno de los objetivos clave de los sistemas educativos es "garantizar una educación inclusiva y equitativa de calidad y promover oportunidades de aprendizaje permanente para todos" (p. 7).

Lo anterior, se traduce en que desde una perspectiva inclusiva todos los estudiantes importan y cada uno tiene el mismo nivel de importancia. Consecuentemente, las diferencias individuales no pueden ser vistas como problemas a ser resueltos, sino como oportunidades para democratizar y enriquecer la enseñanza y aprendizaje (UNESCO, 2017a). En los países latinoamericanos, el desafío de la inclusión educativa es especialmente importante si se considera la elevada inequidad y la deuda existente en términos de calidad. Por ende, los Estados de la región han expresado su reconocimiento al rol central del profesorado en el logro de una educación equitativa y de calidad, y han manifestado su compromiso con el fortalecimiento de los programas de formación docente y la generación de condiciones para un ejercicio profesional de excelencia (UNESCO, 2017b).

Desde un enfoque global, las investigaciones han destacado la relevancia que posee el profesorado como agente en la transformación de las escuelas y los sistemas educativos desde una perspectiva inclusiva (Pantic y Carr, 2017). Sin embargo, la investigación en el contexto chileno ha mostrado que la mayoría de los profesores experimenta dificultades para diseñar e implementar estrategias de enseñanza flexibles, acorde a la heterogeneidad del estudiantado (Jiménez y Montecinos, 2018). Incluso algunos de ellos aún sostienen creencias acerca de la educación que les dificulta reconocer la diversidad como parte de la naturaleza humana y su valoración como eje fundamental del proceso de enseñanza aprendizaje (Apablaza, 2014).

El limitado desarrollo de competencias pedagógicas para una educación inclusiva, ha llevado a cuestionar la pertinencia y efectividad de las experiencias de la formación inicial del profesorado. En este sentido, se ha discutido la importancia de contar con planes de estudio que preparen de manera efectiva a los profesores en formación para una educación inclusiva (San Martín et al., 2017), así como formadores de profesores que contribuyan a ampliar la perspectiva educativa de los futuros docentes y a desarrollar sus competencias para una enseñanza inclusiva (Symeonidou, 2017).

La presente investigación se centra en el estudio de las competencias y estrategias que poseen los formadores de profesores para responder al desafío de la diversidad. Paradójicamente, aunque se ha reconocido la importancia de su rol en una educación inclusiva, existe un grupo sumamente reducido de trabajos en esta área (OECD, 2010). Dentro de ellos, Forlin y Nguyet (2010) en una investigación realizada en Vietnam, encontraron que los formadores de profesores están escasamente preparados para asumir el rol de educar a los futuros docentes en relación a la inclusión; y destacan la importancia de enfocarse en entrenar a los formadores como requisito para implementar de manera exitosa planes de formación inicial para una educación inclusiva. 
Considerando la escasez de trabajos sobre esta temática, la siguiente investigación aborda el estudio de las competencias y estrategias de formadores de profesores chilenos desde una perspectiva de educación inclusiva. Lo anterior, con la finalidad de ofrecer antecedentes que permitan avanzar en la comprensión de su rol en el desarrollo de competencias de enseñanza inclusiva en los profesores en formación, así como de orientar las iniciativas de perfeccionamiento docente que realizan las universidades en este ámbito.

Con este fin nos hemos planteado las siguientes preguntas de investigación:

- ¿Cuán preparados se perciben los formadores de profesores chilenos en las competencias docentes que favorecen una educación inclusiva?

- ¿Cuáles son las estrategias de enseñanza y apoyo a los estudiantes, basadas en una pedagogía inclusiva, que implementan con mayor frecuencia los formadores de profesores chilenos?

\section{El desafío de la inclusión para la práctica docente}

Desde hace algunas décadas, la perspectiva de educación inclusiva ha permitido el análisis de los desafíos que implica la equidad en la educación escolar y ha abordado el mandato que tienen los Estados de transformar los sistemas educativos para que todos los estudiantes, sin restricciones, tengan oportunidades equiparables y de calidad que garanticen el ejercicio pleno del derecho a la educación (Echeita, 2017).

Sin embargo, esta perspectiva también ha dejado entrever la complejidad que conlleva la transformación de las instituciones educativas, considerando la diversidad de retos a los que estas se enfrentan y el dinamismo de los sistemas educativos. Las escuelas están llamadas a ampliar la presencia, la participación y el aprendizaje del estudiantado, al tiempo que trabajan en minimizar o eliminar las barreras, instaladas en distintos niveles de la institución, que impiden el logro de una educación sin exclusiones para todos (Echeita, 2013).

En este escenario complejo, se ha destacado la importancia que tiene el profesorado en la transformación de las aulas y los centros educativos (Durán y Giné, 2011), esperando que puedan contribuir en la construcción de una cultura de centro basada en los valores de la inclusión, participar en la implementación de políticas escolares de apoyo al estudiantado y liderar actividades de enseñanza y aprendizaje que respondan a la diversidad (Booth y Ainscow, 2011). Sin embargo, estos desafíos suelen ser una tarea difícil para el profesorado, especialmente si se considera que las políticas educativas se transforman velozmente y que no ha existido una comprensión compartida acerca de lo que constituye una buena práctica docente en materia de educación inclusiva (Rouse, 2008).

En este contexto, ha surgido el concepto de pedagogía inclusiva, que pone de relieve la importancia del quehacer del profesorado en la reducción de las desigualdades en el aprendizaje y en la mejora de la calidad de educación (Florian, 2015). Este enfoque aboga por el enriquecimiento de los entornos educativos y la generación de oportunidades de aprendizaje para todo el estudiantado, a partir del diseño de situaciones de aprendizaje variadas y flexibles, que les brinde la oportunidad de elegir (Florian y Black-Hawkings, 2011; Florian y Linklater, 2010).

Sin embargo, pese a la importancia que se le reconoce al rol docente, el análisis de la investigación internacional ha evidenciado que, en la actualidad, el profesorado no está 
suficientemente preparado para lidiar con el amplio rango de diversidad presente en las escuelas (Spratt y Florian, 2013).

En el contexto chileno, se ha visto que las barreras para la inclusión asociadas a la práctica docente, se encuentran instaladas en las concepciones de los profesores y en las estrategias que implementan en aula. Al respecto, el profesorado suele tener una perspectiva individual basada en un modelo médico/rehabilitador, que entiende las dificultades de aprendizaje como surgidas exclusivamente de los déficits del estudiantado (Muñoz, López y Assaél, 2015). Asimismo, el profesorado posee una percepción contradictoria de la diversidad, ya que pese a valorarla como una condición inherente al ser humano, también la consideran un obstáculo para la práctica docente (Apablaza, 2014).

Por su parte, la conceptualización del profesorado acerca de la diversidad, influye en las estrategias empleadas para la gestión del aula. En un estudio con profesores chilenos, Jiménez y Montecinos (2018) encontraron que predomina un discurso en que la diversidad es comprendida como una diferencia que surge de algún déficit del estudiantado y que, en consecuencia, desarrollan un estilo de enseñanza homogéneo "donde las actividades, metodologías, formas de participación, niveles de exigencia, así como formas de evaluación se realizan bajo una modalidad única para todos los estudiantes" (p.11).

Considerando la complejidad de una pedagogía inclusiva, abordar su desarrollo desde el marco de las competencias implica comprender que el profesorado debería poseer determinadas concepciones educativas, comprender ciertos hechos del proceso de enseñanza aprendizaje y ser capaz de actuar con eficacia en su práctica docente para promover una educación inclusiva (Echeita, 2015). En este sentido, la formación de profesores es la responsable de contribuir al logro de una comprensión más profunda de los problemas sociales y educativos que pueden afectar el aprendizaje; así como de un rango más amplio de estrategias para apoyar al alumnado y enfrentar estas dificultades (Rouse, 2008).

En un trabajo acerca de esta temática, la Agencia Europea para el Desarrollo de la Educación del Alumnado con Necesidades Educativas Especiales (2012) logró identificar cuatro áreas de competencias en las que debe formarse todo el profesorado: valorar la diversidad del alumnado, entendiendo las diferencias entre estudiantes como un recurso educativo; apoyar a todo el alumnado, a partir de expectativas elevadas respecto de todos los estudiantes; trabajar en equipo, colaborando con otros profesionales del centro y con las familias; y perfeccionar las habilidades profesionales de manera permanente, asumiendo la responsabilidad de aprender a lo largo de la vida.

\section{La formación de profesores para una educación inclusiva}

Considerando la importancia de la formación inicial del profesorado en el desarrollo de las competencias recién descritas, una serie de investigaciones han intentado caracterizar los modos en que esta se aborda en distintos países y su efectividad.

En relación a esto, se ha visto que los planes de formación del profesorado consideran la inclusión desde distintos enfoques. La mayoría lo ha desarrollado desde un enfoque de unidades individuales, a partir de seminarios o talleres de corta duración. Con menos frecuencia, se ha abordado mediante el trabajo asociado entre la universidad y la escuela, a partir de experiencias prácticas que permiten comprender la complejidad de la educación inclusiva en contexto. Finalmente, en casos particulares, la educación inclusiva se ha 
transformado en la columbra vertebral de la formación de profesores, permeando todas las asignaturas con sus contenidos (Symeonidou, 2017).

El análisis de la efectividad de estas aproximaciones ha mostrado una contribución variable al desarrollo de las competencias requeridas para la educación inclusiva. En general, se ha visto que las experiencias de aprendizaje en este ámbito poseen un efecto positivo en el desarrollo de actitudes, conocimientos y habilidades necesarias para una pedagogía inclusiva (Kurniawati et al., 2014). Igualmente, se ha encontrado que dicha formación es más efectiva si considera un periodo extenso que combina varias unidades de aprendizaje, si permite comprender la educación inclusiva desde una perspectiva contextualizada y si cuestiona las nociones tradicionales de la educación especial, a partir del contacto directo con el mundo escolar y la retroalimentación del desempeño de los futuros profesores (Durán y Giné, 2011; Kurniawati et al., 2014; Symeonidou, 2017).

En el contexto nacional la investigación, a pesar de ser incipiente, ha dejado entrever las limitaciones de la formación inicial del profesorado para la inclusión. Al respecto, Tenorio (2011), encontró que la mayoría del estudiantado indica no haber recibido formación en las temáticas de diversidad, integración escolar y necesidades educativas especiales, y no se siente preparado para la inclusión, lo que atribuyen al excesivo foco de sus carreras en el saber disciplinar, en desmedro del saber pedagógico. Asimismo, un análisis de los programas de formación realizado por San Martín y otros (2017) encontró que las temáticas referidas a la educación inclusiva solían tratarse en asignaturas específicas, con un enfoque basado en el discurso, más que en la implementación de prácticas pedagógicas concretas.

Los antecedentes recién descritos ponen de relieve la importancia de fortalecer la formación inicial del profesorado en materia de educación inclusiva, atendiendo tanto a los planes de estudio como al rol de los formadores del profesorado. En relación a esto último, asumir el rol formador desde una perspectiva inclusiva, implica no solo adquirir nuevas competencias docentes, sino superar las barreras instaladas en las instituciones universitarias. La investigación en este contexto, ha mostrado que, al igual que en las escuelas, la mayoría del cuerpo docente conciben la diversidad del estudiantado desde la perspectiva del déficit y desatienden su responsabilidad de generar condiciones para que estos progresen en sus carreras, al considerar que son los estudiantes quienes deben adaptarse a la universidad y asumir de manera individual la responsabilidad por sus logros (O'Shea et al., 2015).

En consecuencia, el logro de una pedagogía inclusiva se ha transformado también en un desafío para las instituciones universitarias y en especial, si se trata de los docentes que forman al profesorado. La investigación en el contexto universitario, indica que en la educación superior una pedagogía inclusiva debe flexibilizar y diversificar el proceso de enseñanza aprendizaje, otorgando oportunidades al estudiantado para elegir entre variadas situaciones y recursos formativos según sus necesidades e intereses (Devlin y O'Shea, 2012; Gavira y Moriña, 2015).

Este enfoque también compromete al cuerpo docente con el conocimiento del estudiantado, con el diálogo continuo acerca del valor de la diversidad en la sociedad, así como con el apoyo académico y emocional que necesitan todos los estudiantes para enfrentar la formación universitaria (Devlin y O'Shea, 2012; Hockings, Brett y Terentjevs, 2012; Hughes, Corcoran y Slee, 2016). Además, los formadores universitarios deben avanzar hacia el diseño de experiencias de aprendizaje universales, prácticas y 
participativas, que integren las tecnologías para favorecer el compromiso con la formación y los logros de aprendizaje (Moriña, Cortés y Molina, 2015); así como el trabajo colaborativo entre estudiantes para la construcción colectiva del conocimiento y el desarrollo del apoyo mutuo (Barkley, Cross y Major, 2007; Zheng, Niiya y Warschauer, 2015).

La presente investigación recoge los aportes del enfoque de la pedagogía inclusiva en la educación universitaria y los retos asociados a la formación de profesores, como marcos de referencia para el estudio de las competencias y estrategias que emplean los docentes universitarios de las carreras de pedagogía chilenas.

\section{Método}

El presente artículo presenta dos estudios cuantitativos complementarios, basados en un diseño descriptivo, de corte transversal. En el primero, nos interesaba abordar la percepción que tienen los formadores de profesores acerca de su preparación en competencias docentes, poniendo especial atención en aquellas habilidades relevantes para el desarrollo de una educación inclusiva. En el segundo estudio, nos importaba profundizar en aquellas estrategias más específicas que implementan los formadores de profesores y que son propias de una pedagogía inclusiva.

\section{Participantes}

Ambos estudios se desarrollaron con docentes pertenecientes a instituciones de educación superior chilenas. En el primer estudio participaron 67 formadores de profesores de una universidad pública de la Región Metropolitana. De estos el 34\% son mujeres y $66 \%$ hombres, y presentan una edad promedio de 46 años, siendo el mínimo 25 y el máximo 76. Con relación a su carrera académica, los formadores tienen en promedio 14 años de experiencia en docencia universitaria, con un mínimo de 1 año y un máximo de 43. Del total $19 \%$ posee grado de licenciado, 39\% de magíster y $42 \%$ de doctor.

En el segundo estudio, participaron 76 formadores de profesores, que pertenecen a siete universidades, tanto públicas como privadas, emplazadas en tres regiones del país: Metropolitana, del Maule y del Bío Bío. De los participantes, $44 \%$ son mujeres y $56 \%$ hombres; en promedio tienen 46 años de edad, siendo el mínimo 24 y el máximo 73. En lo académico, estos poseen 15 años de experiencia promedio, con un mínimo de 1 año y un máximo de 49 . Asimismo, el $15 \%$ posee grado de licenciado, $61 \%$ de magíster y $24 \%$ de doctor.

En ambos estudios, se empleó muestreo no probabilístico, intencionado, siguiendo un criterio de conveniencia, sobre la base de la voluntariedad expresada por los docentes y su participación en proyectos institucionales de fortalecimiento a las carreras de pedagogía desarrollados por las universidades a las que pertenecían.

\section{Variables e instrumentos}

Competencias docentes percibidas, la percepción de competencias para la docencia universitaria corresponde a la apreciación que tienen los académicos acerca de su grado de preparación en las habilidades requeridas para el desarrollo de una docencia efectiva (Torra et al., 2012). Esta variable fue valorada en el primer estudio, mediante la Escala de Competencias para la Docencia Universitaria, creada en esta investigación, a partir de las dimensiones e indicadores propuestos por Torra y colaboradores (2012) y ante la ausencia 
de otro instrumento validado en el contexto nacional que considere tal diversidad de competencias.

La Escala corresponde a un instrumento estructurado, que presenta 48 afirmaciones que describen habilidades propias de una docencia universitaria efectiva. Ante cada afirmación, los docentes deben seleccionar entre cuatro alternativas de respuesta, redactadas en formato Likert ("nada preparado", "poco preparado", "bastante preparado", "muy preparado").

El estudio de las características psicométricas de la escala, se realizó con una muestra de 225 docentes universitarios, pertenecientes a distintas áreas de formación de la misma universidad en que fue realizado el primer estudio. Dicho análisis permitió identificar una estructura similar a la descrita en la teoría y una apropiada consistencia interna de las distintas competencias. Considerando el tipo de variable estudiada, la indagación de estructura de la Escala se realizó mediante el Análisis Factorial Exploratorio, utilizando matrices policóricas (Domínguez, 2014). En concreto, se extrajeron los factores por medio del método de Cuadrados Mínimos Diagonales Ponderados (WLSMv) y el método de rotación Geomin, útiles para el análisis de datos que no cumplen con supuestos de normalidad (Lloret-Segura et al., 2014).

$\mathrm{El}$ análisis de estructura permitió determinar que las competencias evaluadas pueden ser descritas como subescalas separadas, las que se describe a continuación:

Competencia de planificación y gestión de la docencia, que refiere a las capacidades de los docentes para diseñar, orientar y desarrollar contenidos, actividades, evaluaciones y materiales vinculados al proceso de enseñanza y aprendizaje. Esta subescala se estructura en un único factor, compuesto por 6 ítems, que mostró un adecuado ajuste en términos de su estructura $\left(X^{2}=21,0, p=0,01 ; \mathrm{CFI}=0,99 ; \mathrm{TLI}=0,99\right.$; SRMR $\left.=0,04\right)$ y una apropiada consistencia interna $(\omega$ de McDonald $=0,87)$.

Competencia metodológica, que implica las capacidades docentes para desarrollar estrategias de aprendizaje, enseñanza y evaluación, coherentes con las necesidades del estudiantado y los resultados curriculares esperados. Esta sub-escala se organiza en un único factor, compuesto por 10 ítems, que ha evidenciado una estructura ajustada $\left(X^{2}=127,7, p<0,001\right.$; CFI $=0,98 ;$ TLI $=0,97 ;$ y SRMR $=0,06)$ y una consistencia interna adecuada $(\omega$ de McDonald $=0,90$ ).

Competencia interpersonal, que corresponde a las capacidades docentes para promover la valoración de la diversidad, la atención a las motivaciones y necesidades de los estudiantes y la creación de climas de aula basados en la empatía. Esta sub-escala posee una estructura de dos factores que presenta un apropiado ajuste $\left(X^{2}=27,0, p=0,001\right.$; CFI $=0,99$; y TLI $=0,99)$. El primer factor, contempla 4 ítems, posee una consistencia interna adecuada $(\omega$ de McDonald $=0,84)$ y corresponde conceptualmente a la capacidad de reconocimiento del otro, que implica la habilidad del docente para conocer a todos los estudiantes, aceptar y respetar sus particularidades de cada uno y valorar su diversidad (Hirmas y Ramos, 2015). El segundo factor, considera 4 ítems, presenta una apropiada consistencia interna $(\omega$ de McDonald $=0,85)$ y aborda la capacidad empática del docente, es decir, a su capacidad de comprender la perspectiva de los estudiantes y su sensibilidad respecto de los afectos que estos experimentan (Parchomiuk, 2018). 
Competencia comunicativa, que alude a las capacidades docentes para abordar, desde una perspectiva bidireccional, contextualizada y efectiva, la comunicación con los estudiantes. Esta sub-escala se organiza en un factor, que considera 8 ítems, que posee un adecuado ajuste en términos de su estructura $\left(X^{2}=121,6, p<0,001\right.$; CFI $=0,98$; TLI $=0,97$; y SRMR $=0,06)$ y una apropiada consistencia interna $(\omega$ de McDonald $=0,91)$.

Competencia de innovación, que refiere a las capacidades docentes para crear y/o aplicar nuevos conocimientos, perspectivas, estrategias y recursos que contribuyen a la mejora de la enseñanza y el aprendizaje. Esta sub-escala se organiza en un único factor, que integra 8 ítems, que presenta una estructura coherente $\left(X^{2}=156,7, p<0,001\right.$; CFI $=0,97$; TLI $=$ 0,96; $\mathrm{y}$ SRMR $=0,06)$ y una consistencia interna adecuada $(\omega$ de McDonald $=0,92)$.

Competencia de trabajo en equipo, que refiere a la capacidad de los docentes para colaborar con sus pares, en espacios de trabajo donde los equipos asumen colectivamente el compromiso por el logro de objetivos comunes, en atención a los recursos disponibles. Esta sub-escala se estructura en un factor, que considera 7 ítems, que posee una estructura apropiada $\left(X^{2}=101,2, p<0,001 ; \mathrm{CFI}=0,99\right.$; TLI $=0,98 ; \mathrm{y}$ SRMR $\left.=0,05\right)$ y una consistencia interna elevada $(\omega$ de McDonald $=0,93)$.

Estrategias de enseñanza inclusiva reportadas, las estrategias de enseñanza inclusiva reportadas corresponden a las prácticas implementadas que los docentes universitarios indican realizar con la finalidad de favorecer la participación y el aprendizaje de todo el alumnado, con especial atención en aquellos estudiantes que tienden a ser marginados o fracasar académicamente Esta variable fue estudiada por medio de la Escala de Prácticas de Enseñanza Inclusiva (EPEI) de Herrera-Seda, Pérez-Salas y Echeita (2016), que explora la frecuencia con que los docentes emplean acciones basadas en una pedagogía inclusiva en las aulas universitarias. La escala corresponde a un instrumento de auto-reporte, en formato Likert, con 5 alternativas de respuesta ("nunca o casi nunca", "con poca frecuencia", "la mitad de las veces", "con mucha frecuencia y "siempre o casi siempre").

La EPEI se compone de 23 ítems, que se organizan tres factores: a) enseñanza para un aprendizaje significativo, que considera estrategias docentes que favorecen que los estudiantes alcancen un aprendizaje significativo y con sentido (8 ítems); b) co-agencia, que alude a las estrategias que buscan involucrar a los estudiantes como socios activos y comprometidos con la mejora del proceso de aprendizaje ( 7 ítems); y c) apoyo para atender a la diversidad, que refiere a las estrategias orientadas a responder a la diversidad de los estudiantes, contribuyendo a superar las barreras que dificultan el acceso, la participación, el aprendizaje y el desarrollo de todos los alumnos (8 ítems) (Herrera-Seda et al., 2016).

La Escala fue validada con 232 docentes chilenos y el análisis de sus características psicométricas evidenciaron que existe un apropiado ajuste en el modelo de 3 factores recién descrito $\left(X^{2}=234, \mathrm{gl}=187, p=0,01\right)$. Asimismo, el análisis de la consistencia interna evidencia su adecuación en todos los factores del instrumento (Alfa de Cronbach fue 0,79 en enseñanza para un aprendizaje significativo, 0,75 para co-agencia y 0,73 en apoyo para atender a la diversidad) (Herrera-Seda et al., 2016).

\section{Procedimiento}

El contacto con los formadores de profesores se realizó de manera directa, con motivo de su participación en actividades asociadas a los proyectos institucionales de mejora a las carreras de pedagogía. Una vez contactados, se les explicó los fines del estudio y se les 
solicitó la firma de un consentimiento informado, donde se exponía información de la investigación y de los principales resguardos éticos asociados. Tras la firma de este consentimiento, los docentes respondieron los instrumentos de manera individual y en una única ocasión. La aplicación duró 20 minutos en la Escala de Percepción de Competencias para la Docencia Universitaria y 10 minutos en la Escala de Prácticas de Enseñanza Inclusiva.

\section{Análisis de datos}

Considerando los objetivos de este trabajo, el análisis de datos se realizó mediante el programa estadístico SPSS versión 20, que permitió el análisis de frecuencias de las respuestas de los formadores de profesores y la estimación de estadísticos descriptivos de centralidad y dispersión.

\section{Resultados}

\subsection{Competencias docentes de los formadores de profesores}

El análisis de las percepciones de los docentes acerca del desarrollo de sus competencias, ha evidenciado que, en general, los formadores de profesores poseen una percepción positiva de su preparación en las distintas dimensiones analizadas (cuadro 1). Al respecto, los docentes consultados refieren la competencia interpersonal como aquella en que se sienten más capacitados, tanto en lo que refiere a la capacidad de reconocimiento del otro $(\mathrm{M}=3,51 ; \mathrm{DE}=0,47)$, como la de empatía $(\mathrm{M}=3,39 ; \mathrm{DE}=0,55)$. Asimismo, señalan encontrarse adecuadamente preparados para trabajar en equipos académicos $(\mathrm{M}=3,40$; $\mathrm{DE}=0,53)$ orientados a la mejora de su práctica.

Por otra parte, los formadores reportan encontrarse medianamente preparados para planificar y gestionar la docencia de manera adecuada $(\mathrm{M}=3,24 ; \mathrm{DE}=0,55)$. En la misma situación se encuentran respecto de sus habilidades metodológicas asociadas a la implementación de las actividades de aprendizaje $(\mathrm{M}=3,29 ; \mathrm{DE}=0,50)$. Finalmente, la dimensión en que indican sentirse menos capaces corresponde a la competencia de innovación $(\mathrm{M}=2,97 ; \mathrm{DE}=0,71)$, que aborda las habilidades para la construcción o aplicación de nuevas perspectivas en la docencia.

Los resultados de la Escala de Competencias para la Docencia Universitaria, dan cuenta de habilidades generales para abordar la enseñanza en educación superior, de modo que sus subescalas nos proveen de información limitada acerca de la preparación docente para una educación inclusiva. Con este fin y basándonos en los conceptos disponibles en la literatura, hemos definido seis dimensiones transversales que abordan distintos tipos habilidades que reconocidas con anterioridad como propias de una pedagogía inclusiva.

Cuadro 1. Descripción de las competencias docentes de los formadores de profesores

\begin{tabular}{lccccc}
\hline \multicolumn{1}{c}{ COMPETENCIAS } & N & M & DE & MIN & MAX \\
\hline Planificación de la docencia & 67 & 3,24 & 0,55 & 2,00 & 4,00 \\
Metodológica & 67 & 3,29 & 0,50 & 1,90 & 4,00 \\
Interpersonal-Reconocimiento & 67 & 3,51 & 0,47 & 2,50 & 4,00 \\
Interpersonal-Empatía & 67 & 3,39 & 0,55 & 2,25 & 4,00 \\
Innovación & 67 & 2,97 & 0,71 & 1,00 & 4,00 \\
Trabajo en equipo & 67 & 3,40 & 0,53 & 2,00 & 4,00 \\
\hline Fuente:
\end{tabular}

Fuente: Elaboración propia. 
En la figura 1 presentamos un esquema que sintetiza las dimensiones identificadas y los ítems vinculados a estas, considerando la subescala a la que pertenecen.

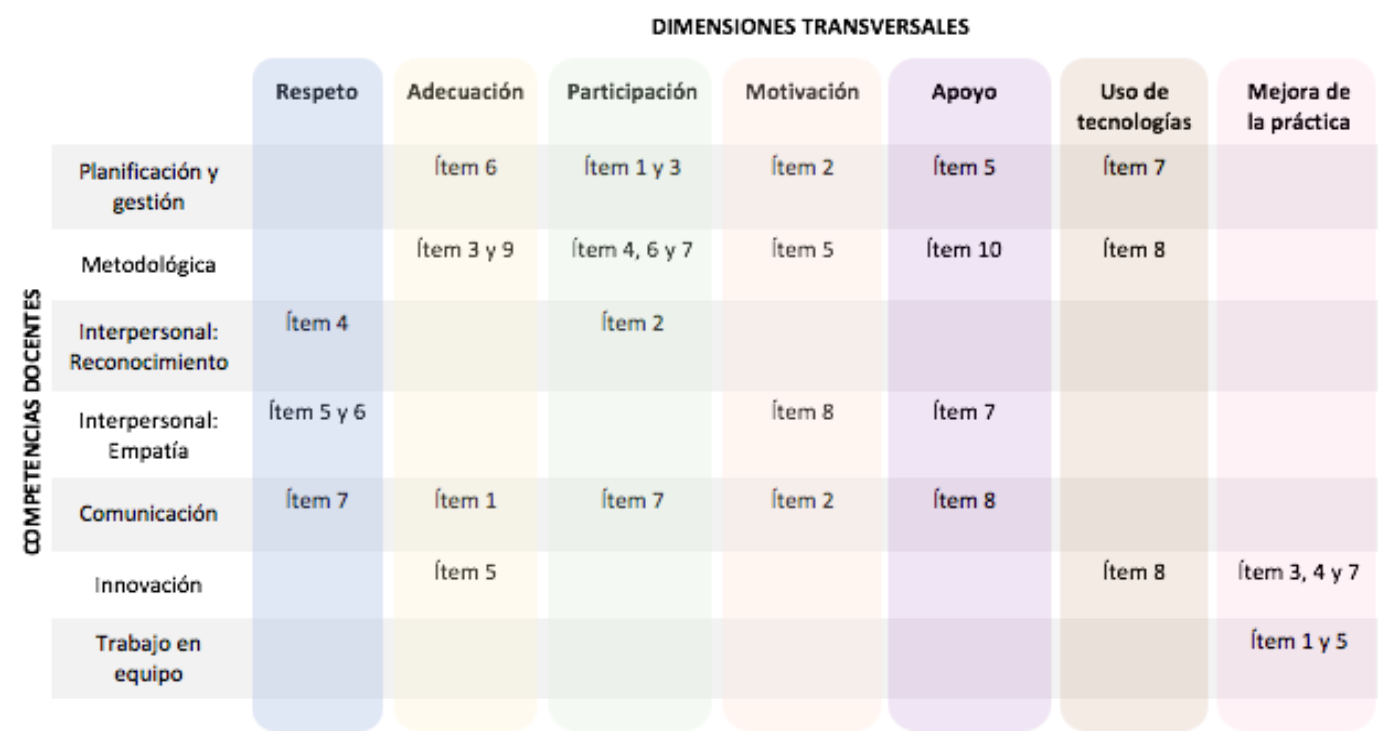

Figura 1. Dimensiones transversales de competencias docentes para una pedagogía inclusiva

Fuente: Elaboración propia.

Desde una perspectiva inclusiva la formación de profesores debe garantizar y promover el respeto por la otra persona (Hirmas y Ramos, 2015). Al respecto, la mayor parte de los formadores de profesores indica sentirse preparado para promover en el estudiantado el respeto por el punto de vista del otro (98\%), generar un clima de empatía en el aula $(95 \%)$ y promover la escucha y la comprensión entre los futuros profesores (94\%). Una menor proporción de estos se considera capacitado para favorecer la valoración de la diversidad en el proceso de enseñanza y aprendizaje (73\%).

Avanzar hacia una formación más flexible constituye un imperativo para una educación inclusiva (Florian y Linklater, 2010) y, en este marco, los formadores de profesores deben ser capaces de adecuar sus estrategias a las características particulares del estudiantado. Al respecto, todos ellos indicaron ser hábiles para expresarse de modo que el estudiantado pueda comprenderlos (100\%). Además, la mayoría indicó poseer habilidades para adaptar la planificación a los logros del alumnado a lo largo del proceso (95\%) y para implementar evaluaciones diversas a los estudiantes evidenciar lo que han aprendido (90\%). Por otro lado, un grupo más acotado de docentes estima estar capacitado para adecuar las innovaciones recogidas de la literatura a su contexto $(81 \%)$ y para adaptar las estrategias y actividades de aprendizaje a las características de sus estudiantes (73\%).

Una de las herramientas que puede contribuir significativamente flexibilizar la formación corresponde al uso de tecnologías para el aprendizaje, que favorecen la accesibilidad y el ajuste de las actividades de aprendizaje (Moriña et al., 2015). Al respecto, un número elevado de formadores percibe estar preparado para utilizarlas en la docencia $(90 \%)$, pero esta proporción se reduce cuando se refiere a la capacidad para diseñar experiencias de aprendizaje que las integren (67\%) y para actualizarse en el uso de nuevas tecnologías $(64 \%)$. 
Promover la participación de los estudiantes también es un elemento clave de una formación inclusiva (Echeita, 2013) y la mayoría de los formadores de profesores se perciben preparados en este desafío. Al respecto, la generalidad de los docentes indica estar habilitados para promover entre los profesores en formación la interacción (94\%) y el diálogo (94\%), así como el desarrollo del pensamiento crítico (97\%). Una proporción algo menor de formadores, considera estar preparado para diseñar actividades de aprendizaje que promuevan la participación de los estudiantes (90\%) y la colaboración con ellos (90\%); y un porcentaje más reducido señala ser capaz de favorecer la participación durante la implementación de las actividades de aprendizaje (73\%).

La perspectiva inclusiva reconoce la diversidad de modos en que cada persona se compromete con la formación (Echeita, 2013) y por ende los docentes deben favorecer la motivación por el aprendizaje en todos los estudiantes. Al respecto, casi la totalidad de formadores expresó sentirse capacitado para explicar a sus estudiantes el sentido de las actividades de aprendizaje (98\%) y la mayoría de ellos expresó su capacidad para establecer relaciones con el estudiantado promueven la motivación por aprender (91\%), para diseñar actividades de formación que favorezcan el compromiso con la formación (88\%) e implementar dichas actividades (87\%).

Por otra parte, enseñar para la diversidad de los estudiantes, implica reconocer que durante la formación todos los alumnos pueden presentar dificultades para participar o aprender (Hughes, Corcoran y Slee, 2016). En consecuencia, corresponde a los docentes desarrollar estrategias de apoyo que permitan al estudiantado alcanzar los objetivos de aprendizaje definidos. Al respecto, la mayoría de los formadores informan sentirse habilitados para promover un clima de confianza que promueva el aprendizaje (95\%), para generar instrucciones claras en las actividades de aprendizaje que facilite su desarrollo $(96 \%)$ y para entregar retroalimentación constructiva luego de las evaluaciones contribuya a la mejora del desempeño $(94 \%)$. Sin embargo, una proporción más pequeña que se considera capaz de diseñar mecanismos de seguimiento de las actividades de formación, acompañando a los estudiantes a través del tiempo (79\%).

Por último, considerar que la inclusión conlleva un proceso de transformación de las instituciones educativas, implica que los profesores deben ser capaces de reflexionar y orientarse para la mejora de su práctica (Booth y Ainscow, 2015). En este punto, la mayoría de los formadores de profesores considera estar preparado para participar en equipos orientados a la mejora educativa (92\%), así como para reflexionar sobre este proceso (92\%). En complemento, un importante grupo indica estar capacitado para implementar innovaciones en su quehacer ( $84 \%$ ) y otro, más reducido, se siente hábil para diseñar innovaciones que contribuyan a mejorar la formación (73\%) o investigar para mejorar el proceso de aprendizaje de todos los estudiantes (66\%) (figura 2).

\subsection{Estrategias de enseñanza inclusiva de los formadores de profesores}

El segundo estudio nos permitió conocer la frecuencia con que los formadores de profesores chilenos emplean estrategias de enseñanza para que todo el estudiantado tenga la posibilidad de acceder la formación y alcanzar los desempeños profesionales requeridos. En este marco analizamos tres dimensiones que agrupan dichas estrategias: enseñanza para un aprendizaje significativo, co-agencia y apoyo para atender a la diversidad. El reporte de cada dimensión se presenta en lo que sigue. 
En general, los docentes indican desarrollar con frecuencia estrategias de enseñanza que contribuyen al logro de aprendizajes significativos $(\mathrm{M}=4,58 ; \mathrm{DE}=0,33)$. El detalle de los ítems de este factor se presenta en la figura 3.

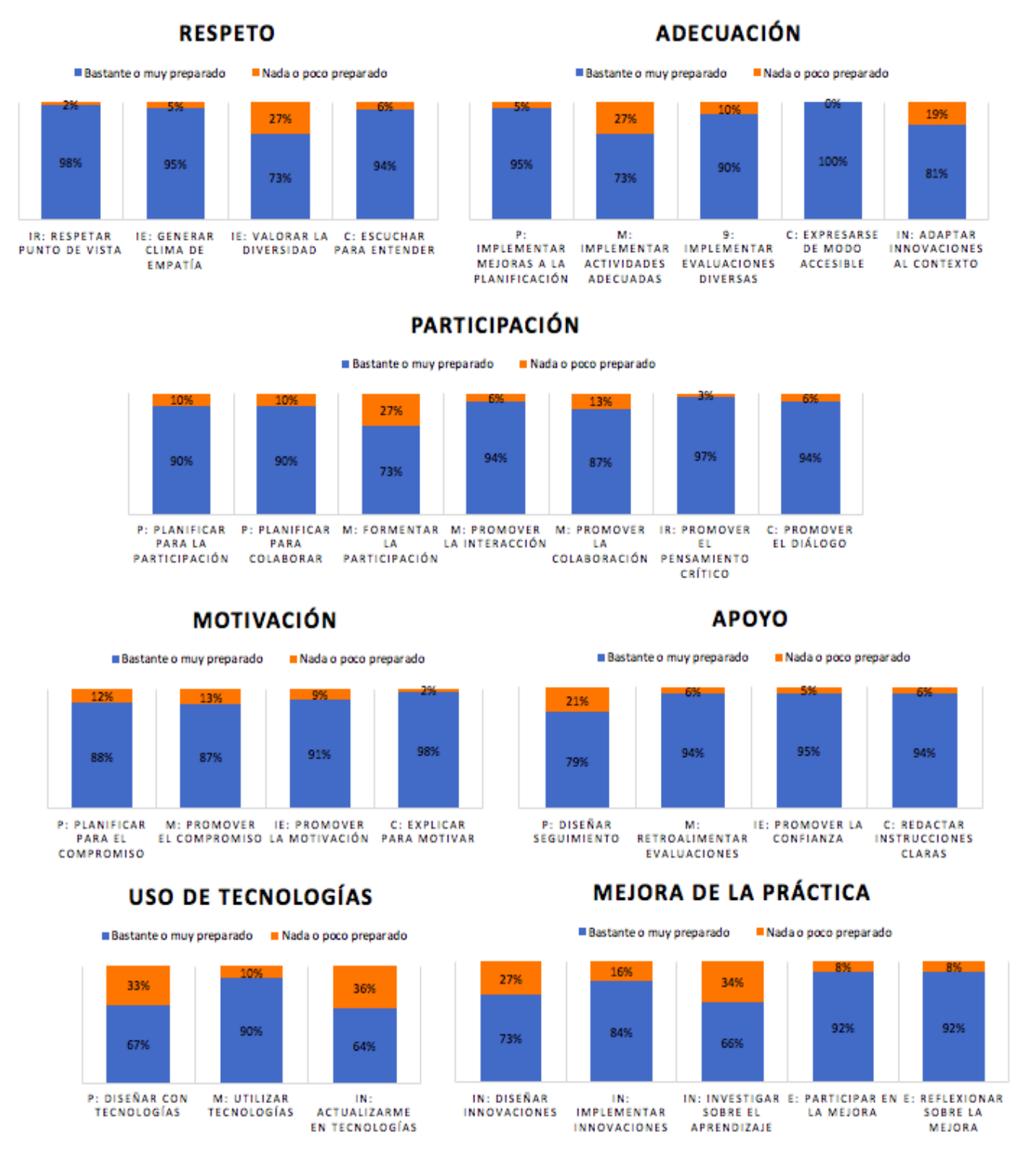

Figura 2. Porcentaje de respuestas en las dimensiones transversales de habilidades docentes para una educación inclusiva

Nota: $\mathrm{P}=$ Planificación y gestión de la docencia; $\mathrm{M}=$ Metodológica; $\mathrm{IR}=$ Interpersonal de reconocimiento del otro; $\mathrm{IE}=$ Interpersonal de empatía; $\mathrm{C}=$ Comunicativa; $\mathrm{IN}=$ Innovación; $\mathrm{E}=$ Trabajo en equipo.

Fuente: Elaboración propia.

El respeto y reconocimiento del otro es un principio relevante de la educación inclusiva y, en este sentido, los formadores indican que con frecuencia dicen a sus estudiantes que deben escuchar las opiniones de sus compañeros sin descalificar $(\mathrm{M}=4,61 ; \mathrm{DE}=0,68)$, pero con una menor regularidad llaman a los alumnos los por su nombre $(\mathrm{M}=4,17$; $\mathrm{DE}=$ $0,97)$ lo que evidencia su conocimiento limitado del estudiantado. 
En relación a la enseñanza, los formadores de profesores expresan implementar con frecuencia experiencias que le permiten a los estudiantes aplicar los contenidos $(\mathrm{M}=4,39$; $\mathrm{DE}=0,77)$; y en menor medida, actividades en que deben cooperar con sus compañeros ( $\mathrm{M}$ $=4,22 ; \mathrm{DE}=0,79)$ o donde pueden desarrollar sus habilidades a partir de tareas variadas $(\mathrm{M}=4,21 ; \mathrm{DE}=0,86)$.

En relación a la evaluación, los docentes señalan que con elevada frecuencia desarrollan distintos tipos de evaluaciones a lo largo de un semestre $(\mathrm{M}=4,48 ; \mathrm{DE}=0,67)$ y con menor regularidad dichas evaluaciones integran distintos tipos de ítems $(\mathrm{M}=4,26 ; \mathrm{DE}=1,02)$.

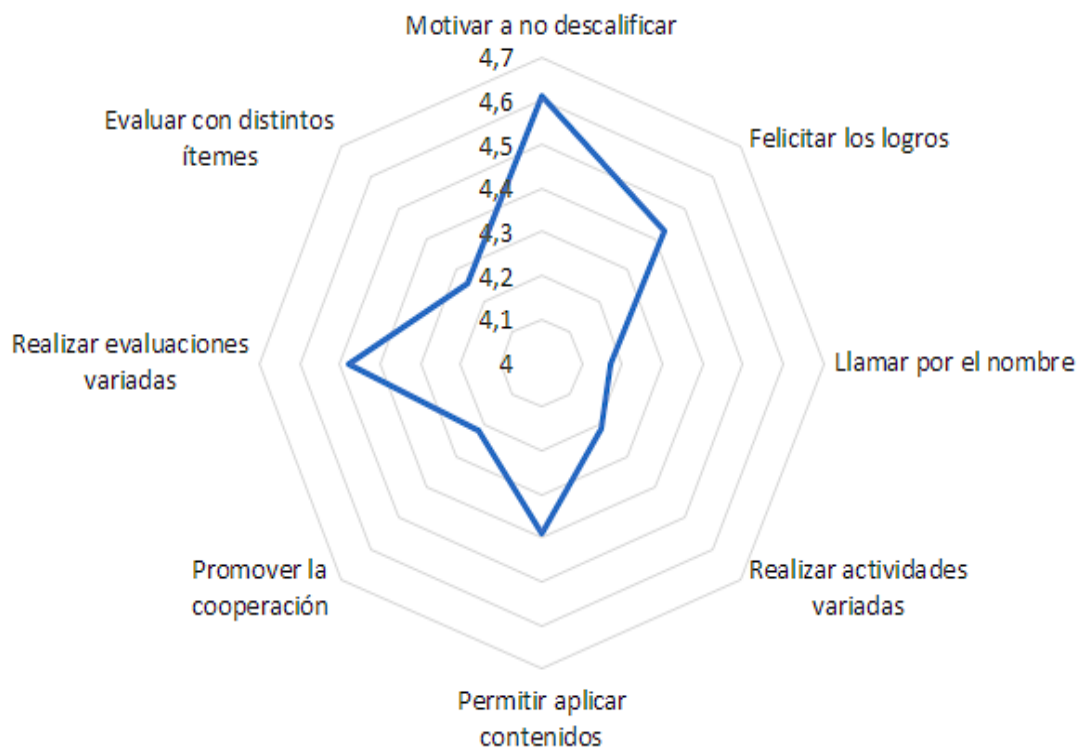

Figura 3. Medias en el uso de estrategias de enseñanza para un aprendizaje significativo Fuente: Elaboración propia.

Por otra parte, la co-agencia corresponde a la dimensión desarrollada con menos frecuencia por formadores de profesores $(\mathrm{M}=4,09 ; \mathrm{DE}=0,51)$. El resultado detallado en sus indicadores se muestra en la figura 4 .

Los formadores de profesores describen el uso habitual de estrategias para fortalecer el aprendizaje, donde la más frecuente es sugerir recursos complementarios para profundizar los conocimientos $(\mathrm{M}=4,43 ; \mathrm{DE}=0,70)$; seguido por dar seguimiento a los logros del estudiantado y reforzar las áreas más débiles $(\mathrm{M}=4,28 ; \mathrm{DE}=0,74)$; y entregar sugerencias concretas para mejorar luego de las evaluaciones $(\mathrm{M}=4,24 ; \mathrm{DE}=0,69)$.

Por el contrario, los formadores desarrollan con menos regularidad acciones para transformar el aula en un espacio de aprendizaje flexible. Comparativamente, modifican la planificación del curso a lo largo del semestre para responder al aprendizaje con menor frecuencia $(\mathrm{M}=3,78 ; \mathrm{DE}=1,05)$ y es aún menos común que entreguen opciones de tareas o trabajos variadas, entre las que el estudiantado puede elegir según su interés $(\mathrm{M}=3,08$; $\mathrm{DE}=1,22)$.

Finalmente, en al apoyo para atender a la diversidad, los formadores de profesores dieron cuenta de uso frecuente de sus estrategias $(\mathrm{M}=4,58$; $\mathrm{DE}=0,33)$, por sobre las otras dimensiones estudiadas. En la figura 5 se presentan de manera pormenorizadas los resultados por ítem. Los formadores de profesores indican que, frecuentemente, informan 
al estudiantado sobre la utilidad que tendrán los aprendizajes de la asignatura cuando sean profesionales $(\mathrm{M}=4,67 ; \mathrm{DE}=0,70)$ y entregan ejemplos adicionales cuando algún estudiante tiene dificultades para comprender $(\mathrm{M}=4,67 ; \mathrm{DE}=0,53)$. Así mismo, reconocen que responden a las dudas del estudiantado fuera de clase $(\mathrm{M}=4,62 ; \mathrm{DE}=0,67)$ y les ayudan a resolver las actividades de la asignatura cuando tienen dificultades $(\mathrm{M}=4,61 ; \mathrm{DE}$ $=0,59)$.

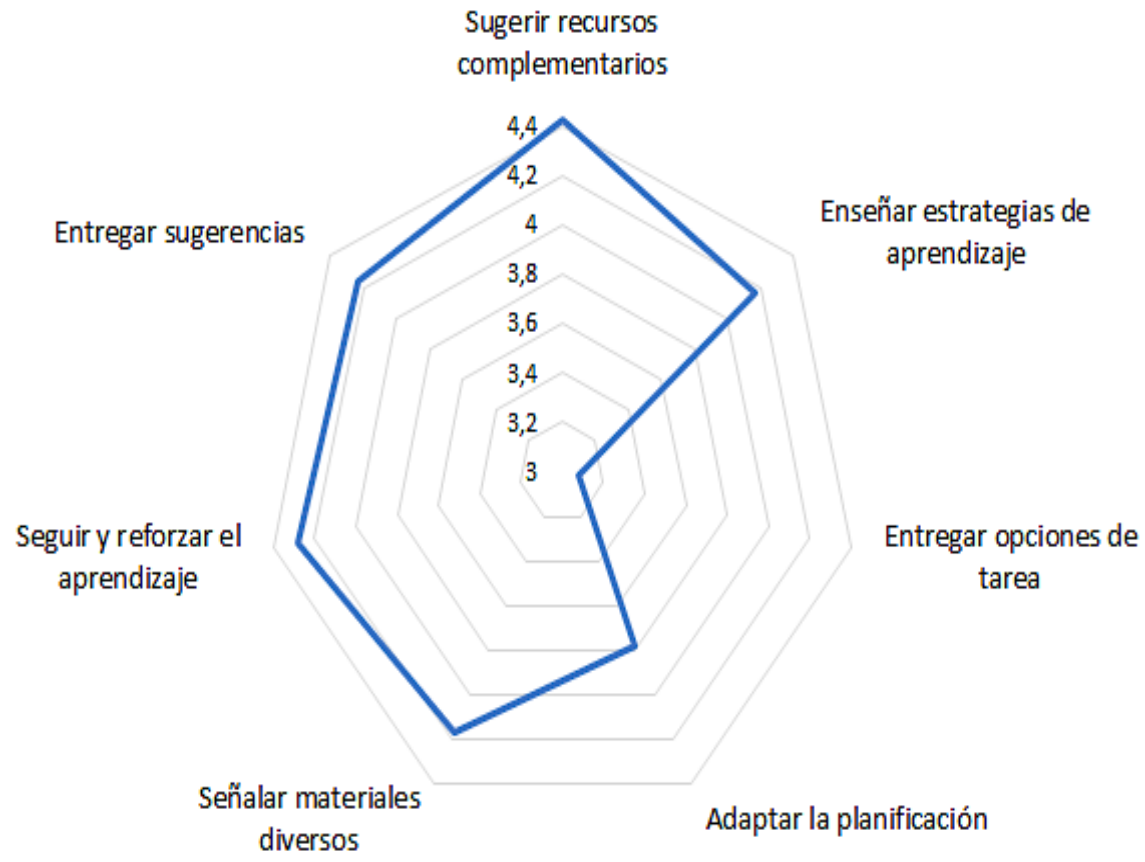

Figura 4. Medias de la frecuencia de uso de estrategias de co-agencia Fuente: Elaboración propia.

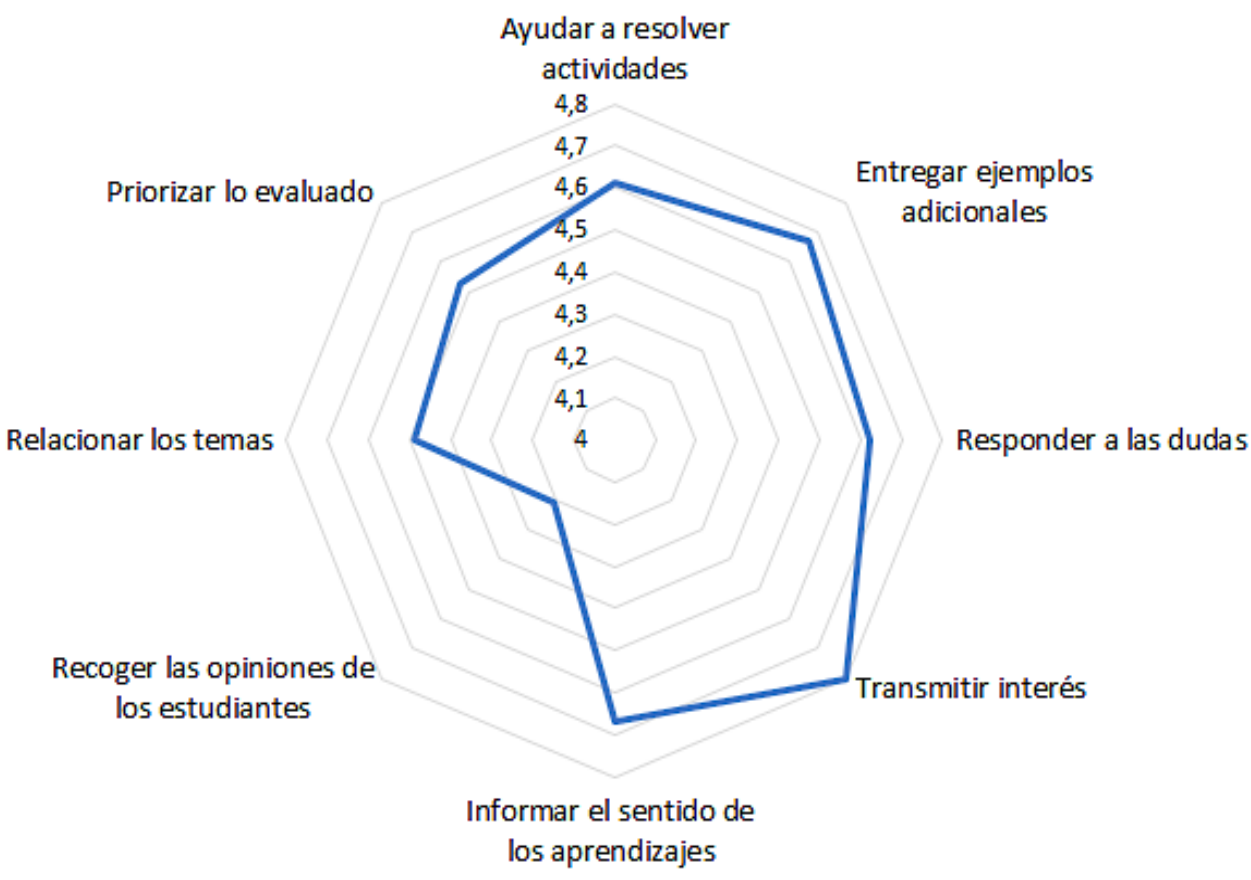

Figura 5. Medias de la frecuencia de uso de estrategias de apoyo para atender a la diversidad 
Fuente: Elaboración propia.

\section{Conclusiones}

Formar al profesorado para una educación inclusiva representa uno de los desafíos fundamentales para lograr el desarrollo de sistemas educativos que garanticen una educación equitativa y de calidad para todo el alumnado. Ello implica transformaciones educativas no solo a nivel escolar, sino también en las instituciones universitarias, las que deben asumir que los formadores de profesores son fundamentales en el desarrollo de las competencias que los futuros profesores necesitarán para liderar una transformación inclusiva en las escuelas (Nye, Konstantopoulos y Hedges, 2004), y que su labor permite a los estudiantes de pedagogía vivenciar, en su propia trayectoria formativa, en qué consiste una educación para todos sin ningún tipo de exclusión (Zapata, 2014)

Sin embargo, las escasas investigaciones conducidas hasta el momento sobre los formadores de profesores (Forlin y Nguyet, 2010), las limitaciones del profesorado en ejercicio (Tenorio, 2010) y la propia experiencia de los estudiantes (Jiménez y Montecinos, 2018) habían indicado la insuficiente capacidad de los formadores de profesores para educar desde una perspectiva inclusiva. Más de estos antecedentes, los resultados de este trabajo mostraron que, en general, el cuerpo académico considera tener ampliamente desarrolladas las competencias para promover una educación inclusiva e implementa con bastante frecuencia estrategias de enseñanza que permiten la participación y el aprendizaje de todo el profesorado en formación, sin ningún tipo de distinción. Las diferencias encontradas en este trabajo deben ser consideradas con cautela, teniendo en cuenta las limitaciones de las escalas de auto-reporte, así como los efectos de deseabilidad social que pueden tener los proyectos de mejoramiento institucional en los que participaban los docentes de la muestra.

Por otra parte, detrás de esta tendencia general alentadora, se encuentran algunos puntos más débiles en las competencias y estrategias del cuerpo académico que forma profesores, tales como el diseño innovaciones en el quehacer docente; la flexibilización y diversificación de las experiencias de aprendizaje; o la incorporación las TIC en el diseño de clases. Lo anterior, pone de manifiesto la relevancia de generar instancias que permitan a los formadores de profesores analizar e innovar acerca de su propia práctica, incorporando enfoques centrados en el aprendizaje de todo el estudiantado, tales como el aprendizaje colaborativo o el diseño universal para el aprendizaje.

Considerando la especificidad de la labor de los formadores de profesores y las limitaciones de esta investigación, es que uno de los desafíos fundamentales que emana de este trabajo es construir escalas más sensibles, que permitan abordar el modo en que estos docentes transmiten una visión y contribuyen al desarrollo de las competencias inclusivas en sus propios estudiantes. De igual manera y más allá de las restricciones propias del autoreporte, la escalas que abordan la pedagogía inclusiva, deben identificar no solo estrategias empleadas para a abordar su quehacer desde una perspectiva inclusiva, sino también las barreras que consciente o inconscientemente desarrollan, limitando la presencia, la participación y el aprendizaje de todos y cada uno de los estudiantes.

Por último, el análisis pormenorizado de los resultados de este trabajo permite orientar el perfeccionamiento docente en las distintas competencias y estrategias requeridas para una formación de profesores con sello inclusivo. En este sentido, el llamado a las instituciones 
universitarias es a contribuir al desarrollo de las competencias que los formadores de profesores requieren para una pedagogía inclusiva, así como de fortalecer su percepción de eficacia respecto a la enseñanza en aulas heterogénea. Lo anterior, en el marco de planes de estudio que abordan la formación para la inclusión de manera profunda, sistemática y contextualizada a los retos de cada sistema educativo y la complejidad de la sociedad actual.

\section{Referencias}

Agencia Europea para el Desarrollo de la Educación del Alumnado con Necesidades Educativas Especiales. (2012). Formación del profesorado para la educación inclusiva. Perfil profesional del docente en la educación inclusiva. Bruselas: Agencia Europea para el Desarrollo de la Educación del Alumnado con Necesidades Educativas Especiales.

Apablaza, M. (2014). Representaciones sociales de profesores respecto de la diversidad escolar en relación a los contextos de desempeño profesional, prácticas y formación inicial. Estudios Pedagógicos, 40(1), 7-24. https://doi.org/10.4067/S0718-07052014000100001

Barkley, E., Cross, K. y Major, C. (2007). Técnicas de aprendizaje colaborativo: Manual para el profesorado universitario. Madrid: Morata.

Booth, T. y Ainscow, M. (2015). Guía para la educación inclusiva: Desarrollando el aprendizaje y la participación en los centros escolares. Madrid: FUHEM.

Devlin, M. y O’Shea, H. (2012). Effective university teaching: Views of Australian university students from low socio-economic status backgrounds. Teaching in Higher Education, 17(4), 385-397. https://doi.org/10.1080/13562517.2011.641006

Domínguez, S. (2014). ¿Matrices policóricas/tetracóricas o matrices Pearson? Un estudio metodológico. Revista Argentina de Ciencias del Comportamiento, 6(1), 39-48.

Durán, D. y Giné, C. (2011). La formación del profesorado para la educación inclusiva: Un proceso de desarrollo profesional y de mejora de los centros para atender la diversidad. Revista Latinoamericana de Educación Inclusiva, 5(2), 153-170.

Echeita, G. (2013). El apoyo entre el alumnado para aprender y participar. En G. Echeita, C. Simón, M. Sandoval y H. Monarca (Eds.), Cómo fomentar las redes naturales de apoyo en el marco de una escuela inclusiva. Propuestas prácticas (pp. 55-76). Madrid: MAD.

Echeita, G. (2015). Competencias esenciales en la formación inicial de un profesorado inclusivo. Un proyecto de la Agencia Europea para el Desarrollo de las Necesidades Educativas Especiales. Tendencias Pedagógicas, 19, 7-24.

Echeita, G. (2017). Educación inclusiva. Sonrisas y lágrimas. Aula Abierta, 46, 17-24. https://doi.org/10.17811/rifie.46.2017.17-24

Florian, L. (2015). Inclusive pedagogy: A transformative approach to individual differences but can it help reduce educational inequalities? Scottish Educational Review, 47(1), 5-14.

Florian, L. y Black-Hawkins, K. (201 1). Exploring inclusive pedagogy. British Educational Research Journal, 37(5), 813-828. https://doi.org/10.1080/01411926.2010.501096

Florian, L. y Linklater H. (2010). Preparing teachers for inclusive education: Using inclusive pedagogy to enhance teaching and learning for all. Cambridge Journal of Education, $40(4)$, 369-386. https://doi.org/10.1080/0305764X.2010.526588 
Forlin, C. y Nguyet, D. (2010). A national strategy for supporting teacher educators to prepare teachers for inclusion. En C. Forlin (Ed.), Teacher education for inclusion. Changing paradigms and innovative approaches (pp. 34-44). Quebec: Taylor \& Francis.

Gavira, R. y Moriña, A. (2015). Hidden voices in higher education: Inclusive policies and practices in social science and law classrooms. International Journal of Inclusive Education, 14(4), 365378. https://doi.org/10.1080/13603116.2014.935812

Herrera-Seda, C. M., Pérez-Salas, C. P. y Echeita, G. (2016). Teorías implícitas y prácticas de enseñanza que promueven la inclusión educativa en la universidad: Instrumentos y antecedentes para la reflexión y discusión. Formación Universitaria, 9(5), 49-64. https://doi.org/10.4067/S07 18-50062016000500006

Hirmas, C. y Ramos, L. (2015). El viaje hacia la diferencia: La escuela inclusiva. Santiago: Ediciones SM.

Hockings, C., Brett, P. y Terentjevs, M. (2012). Making a difference. Inclusive learning and teaching in higher education through open educational resources. Distance Education, 32(2), 237-252. https://doi.org/10.1080/01587919.2012.692066

Hughes, K., Corcoran, T. y Slee, R. (2016). Health-inclusive higher education: Listening to students with disabilities or chronic illnesses. Higher Education Research \& Development, 35(3), 488-501. https://doi.org/10.1080/07294360.2015.1107885

Jiménez, F. y Montecinos, C. (2018). Diversidad, modelos de gestión y formación inicial docente: Desafíos formativos desde una perspectiva de justicia social. Revista Brasileira de Educação, 23, 1-21.

Kurniawati, F., De Boer, A., Minnaert, A. y Mangunsong, F. (2014). Characteristics of primary teacher training programmes on inclusion: A literature focus. Educational Research, 56(3), 310-326. https://doi.org/10.1080/00131881.2014.934555

Lloret-Segura, S., Ferreres-Traver, A., Hernández-Baeza, A. y Tomás-Marco, I. (2014). El análisis factorial exploratorio de los ítems: Una guía práctica, revisada y actualizada. Anales de Psicología, 30(3), 1151-1169. https://doi.org/10.6018/analesps.30.3.199361

Marchesi, A. y Martín, E. (2014). Calidad de la enseñanza en tiempos de crisis. Madrid: Alianza Editorial.

Moriña, A., Cortés, M. D. y Molina, V. (2015). Educación inclusiva en la enseñanza superior: Soñando al profesorado ideal. Revista Latinoamericana de Educación Inclusiva, 9(2), 161-175.

Muñoz Villa, M., López Cruz, M. y Assaél, J. (2015). Concepciones docentes para responder a la diversidad: ¿Barreras o recursos para la inclusión educativa? Psicoperspectivas, 14(3), 68-79.

Nye, B., Konstantopoulos, S. y Hedges, L. V. (2004). How large are teacher effects? Educational $\begin{array}{llll}\text { Evaluation and Policy } & \text { 237-257. }\end{array}$ https://doi.org/10.3102/01623737026003237

OCDE. (2010). Educating teachers for diversity: Meeting the challenge. París: OCDE. https://doi.org/10.1787/9789264079731-en

O'Shea, S., Lysaght, P., Roberts, J. y Harwood, V. (2015). Shifting the blame in higher education, social inclusion and deficit discourses. Higher Education Research \& Development, 35(2), 322336. https://doi.org/10.1080/07294360.2015.1087388

Pantic, N. y Carr, D. (2017). Educating teachers as agents of social justice: A virtue ethical perspective. En L. Florian y N. Pantíc (Eds.), Teacher education for the changing demographics of schooling. Issues for research and practice (pp. 55-66). Zúrich: Springer Nature. https://doi.org/10.1007/978-3-319-54389-5_5 
Parchomiuk, M. (2018). Teacher empathy and attitudes towards individuals with disabilities. International Journal of Disability, Development and Education, 65(5), 1-14. https://doi.org/10.1080/1034912X.2018.1460654

Rouse, M. (2008). Developing inclusive practice: A role for teachers and teacher education. Education in the North, 16(1), 6-13.

San Martín, C., Villalobos, C., Muñoz, C. y Wyman, I. (2017). Formación inicial docente para la educación inclusiva. Análisis de tres programas chilenos de pedagogía en educación básica que incorporan la perspectiva de la educación inclusiva. Calidad en la Educación, 46, 20-52. https://doi.org/10.4067/So7 18-45652017000100020

Spratt, J. y Florian, L. (2013). Applying the principles of inclusive pedagogy in initial teacher education: From university based course to classroom action. Revista de Investigación en Educación, 11(3), 133-140.

Symeonidou, S. (2017). Initial teacher education for inclusion: A review of the literature. Disability E' Society, 32(3), 401-422. https://doi.org/10.1080/09687599.2017.1298992

Tenorio, S. (2011). Formación inicial docente y necesidades educativas especiales. Estudios Pedagógicos, 37(2), 249-265. https://doi.org/10.4067/So718-07052011000200015

Torra, I. de Corral, I., Pérez, M., Triadó, X., Pagès, T., Valderrama, E., ..., y Tena, A. (2012). Identificación de competencias docentes que orienten el desarrollo de planes de formación dirigidos a profesorado universitario. Revista de Docencia Universitaria, 10(2), 21-56. https://doi.org/10.4995/redu.2012.6096

UNESCO. (2015). Declaración de Incheon y marco de acción para la realización del objetivo de desarrollo sostenible 4. París: UNESCO.

UNESCO. (2017a). A guide for ensuring inclusion and equity in education. París: UNESCO.

UNESCO. (2017b). Declaración de Buenos Aires. Buenos Aires: UNESCO.

Zapata, R. (2014). Diversidad cultural en la formación del futuro profesorado en América Latina. Necesidades y perspectivas. Revista Electrónica Interuniversitaria de Formación del Profesorado, 17(2), 219-234. https://doi.org/10.6018/reifop.17.2.197611

Zheng, B., Niiya, M. y Warschauer, M. (2015). Wikis and collaborative learning in higher education. Technology, Pedagogy and Education, 24(3), 357-374. https://doi.org/10.1080/1475939X.2014.948041

\section{Breve CV de los autores}

\section{Carolina García González}

Profesora de Enseñanza Media de Historia, Magíster en Ciencias de la Educación y Doctora en Ciencias de la Educación de la Pontificia Universidad Católica de Chile. Académica del Área de Formación de Profesores, de la Unidad de Innovación Educativa de la Universidad de Santiago de Chile. Investigadora en temáticas de formación ciudadana en el sistema escolar, en relación a la didáctica de la historia y las ciencias sociales, las perspectivas curriculares, la convivencia escolar, y el desarrollo de competencias ciudadanas en la formación inicial y continua de profesores. ORCID ID: https://orcid.org/o000-0002-2723-2624. Email: carolina.garcia.g@usach.cl 


\section{Constanza Herrera-Seda}

Magister en Investigación Social y Desarrollo y Doctora en Psicología, en el área de enseñanza aprendizaje de la Universidad de Concepción. Académica del Área de Formación de Profesores, de la Unidad de Innovación Educativa de la Universidad de Santiago de Chile. Investigadora en temáticas de educación inclusiva, particularmente, en la formación de profesores para una educación inclusiva, las creencias y prácticas docentes para una pedagogía inclusiva, el desarrollo de competencias de los formadores de profesores para una educación inclusiva y estrategias docentes para el aprendizaje para todos en el contex to escolar. ORCID ID: https://orcid.org/0000-0001-5778-6495. Email: constanza.herrera.s@usach.cl

\section{Carlos Vanegas Ortega}

Profesor de Matemáticas y Física de la Universidad de Antioquia (Colombia), Magíster en Educación en la línea de Ciencias Experimentales y Matemáticas de la Universidad de Antioquia, Magíster en Ciencias de la Educación y Doctor en Ciencias de la Educación de la Pontificia Universidad Católica de Chile. Académico del Área de Formación de Profesores, de la Unidad de Innovación Educativa de la Universidad de Santiago de Chile. Investigador en temáticas de didáctica de las ciencias experimentales y matemáticas, formación de profesores: pensamiento reflexivo del profesor, identidad profesional docente, prácticas pedagógicas, inserción e inducción del profesorado principiante. ORCID ID: https://orcid.org/0000-0002-5364-0664. Email: carlos.venegas.o@usach.cl 